\title{
Optimal Input Design Using Generalized Binary Sequence*
}

\author{
JIUN-KAI CHEN $\dagger$ and CHENG-CHING YU†
}

Key Words--Identification; input signal; experiment design; binary sequence; linear system.

\begin{abstract}
The generalized binary sequence (GBS) of Tulleken offers an attractive alternative in input design for system identification. In terms of time-domain responses, the GBS ranges from a square-wave sequence to a step input as the non-switching probability changes from zero to unity. In terms of frequency responses, the emphasis of input spectrum changes from low frequency to the Nyquist frequency as the non-switching probability varies. In this work, analytical expression for the optimal non-switching probability for the GBS is derived when the specification is given on a single-frequency value. Moreover, for a more realistic case, if the specification is given as a frequency interval, the optimal non-switching probability can also be obtained analytically. A linear example is used to illustrate this GBS-based input design procedure. (C) 1997 Elsevier Science Ltd.
\end{abstract}

\section{Introduction}

It is desirable to have an accurate process model for the design of a controller. However, the quality of the process model depends on the type of the controller used and the tuning rule employed. For example, if the Ziegler-Nichols tuning is used, emphasis is placed on the ultimate frequency (c.g. good cstimates of ultimate gain $\left(K_{u}\right)$ and ultimate frequency $\left.\left(\omega_{u}\right)\right)$. And if a static compensator is required, then, the low-frequency behavior of the model is of major importance. Traditional gain margin and phase matgin types of specifications require quality process information at high-frequency range (e.g. frequency range with phase angles between -135 and -180 ), i.e. quality of the model can be examined at the prescribed single-frequency point or frequency range of interest.

Typically, a process model is obtained from system identification. Initially, the plant input is perturbed in a specific manner and a model is approximated from input-output data (Ljung, 1987; Luyben, 1990; Marlin, 1995; Shen et al., 1996). In the identification procedure, the input design plays a deciding role in process excitation (Goodwin and Payne, 1977; Ljung, 1987; Schoukens et al., 1988; Tulleken, 1990; Belforte and Tay, 1993). Two types of input signals are often employed in chemical process control: step input (Luyben, 1990; Cluett and Wang, 1991; Tyreus and Luyben, 1992; Harris, 1995) and pseudorandom binary sequence (PRBS) (Prett et al., 1986; Tulleken, 1990; Luyben, 1990; Bailey, 1995; Chan, 1995; Marlin, 1995).

For the step input, emphasis is placed on the low (zero) frequency and for the PRBS, the input energy is placed equally over the entire frequency range. However, in many occasions, we

${ }^{*}$ Received 18 March 1997; received in final form 2 June 1997. The Published Proceedings of this IFAC Meeting may be ordered from: Elsevier Science Ltd, The Boulevard, Langford Lane, Kidlington, Oxford, OX5 1GB, UK. This paper was recommended for publication in revised form by Editor Peter Dorato. Corresponding author Cheng-Ching Yu. Tel. + 886-2737-6620; Fax + 886-2-737-6644; E-mail ccyu@ch.ntust.edu.tw. $\dagger$ Department of Chemical Engineering, National Taiwan University of Sci. Technol., Taipei 106, Taiwan. are interested in the frequency (or frequency range) other than these two extremes. The concept of the generalized binary sequence (GBS) of Tulleken (1990) offers some light along that direction. Tulleken uses GBS, initially proposed by Cumming (1970) for improved input design in system identification. The GBS has the same configuration as the PRBS except that an additional parameter switching (or non-switching) probability is introduced. Actually, similar approaches are taken to identify non-linear autoregressive models for high-purity distillation columns (Sriniwas et al., 1995; Chien, 1996). The conventional PRBS can be viewed as the GBS with a switching probability of 0.5 (e.g. tossing a fair coin) and the step input is equivalent to the GBS with a switching probability of zero (e.g. tossing a coin always showing head or tail). More importantly, the GBS can be specified with a switching probability ranging from zero to one. Therefore, it constitutes the basic means for input design in system identification.

The objective of this work is to derive analytical relationships between the optimal switching probability and the frequency of interest. Analytical expressions for both a signal-frequency point and a frequency interval are derived. The remaining of this paper is organized as follows: Section 2 describes the GBS and relevant properties. In Section 3, main results are given and an input design procedure is summarized. A transfer function example is used to illustrate the input design followed by the conclusion.

\section{Generalized binary signal}

2.1. Definition. Consider a sequence of input signal, i.e. $u_{k}, k=1,2,3, \ldots$, with a basic switching (or sampling) time of $T$. The input is switched at two levels: $u_{k}=+h$ or $u_{k}=-h$. At time $k t$, the input $u_{k}$ is related to the previous input signal, $u_{k-1}$, in the following probabilistic manner:

$$
P\left(u_{k}=u_{k-1}\right)=0.5,
$$

where $P(\cdot)$ is the probability for the indicated event and in this case the non-switching probability is: 0.5 . This is the conventional binary signal (BS) as shown in Fig. 1 (i.e. $p=0.5$ ). For a finite number of input, i.c., $u_{k}, k=1, \ldots, N$, this sequence is often referred to as pseudo-random binary signal (PRBS) (Eykhoff, 1974; Goodwin and Payne, 1977; Luyben, 1990) and it is generally regarded as an "optimal" input signal for the reason that frequencies are tested with almost equal power.

It is well known that in system identification one ought to place the energy of the input sequence on the relevant frequency range (Goodwin and Payne, 1977; Ljung, 1987). Instead of extracting relevant information from a poor designed experiment (e.g. using only the initial response from a step test), one would rather improve the input design. The generalized version of the binary signal (GBS) of Tulleken (1990) serves the right purpose.

Instead of fixing the probability at 0.5 , the GBS uses the non-switching probability, $p$, as a design variable, i.e.

$$
P\left(u_{k}=u_{k-1}\right)=p
$$




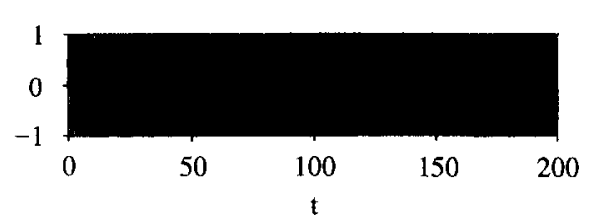

$\mathrm{p}$
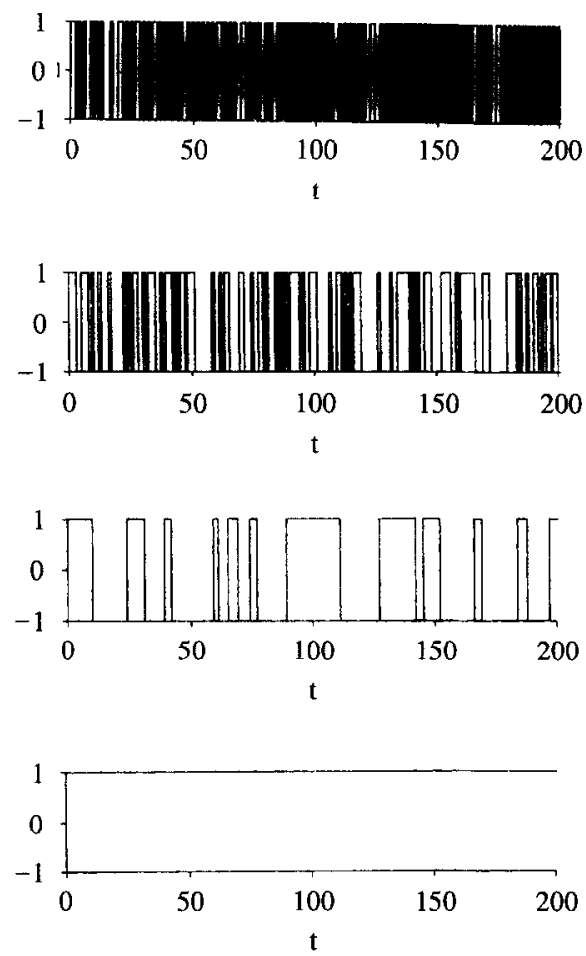

Fig. 1. Test signal of GBS for different values of $p$ with $T=1$.

with $p$ ranging from 0 to $1(0 \leq p \leq 1)$. In doing this the energy of the input signal is no longer equally placed (as what the BS does). More importantly, we can put the emphasis on the frequency range of interest by selecting an appropriate $p$. Figure Fig. 1 shows the input sequence starts from a square wave to a step test as the non-switching probability changes from 0 to 1 .

2.2. Frequency response. Tulleken (1990) derives the frequency spectrum of the GBS. Briefly, it begins with relating the covariance function of the sequence $u$ at time $k$ to that of the previous sampling instance, i.e. for $u_{k}$ and $u_{i}$ with $k>l$,

$$
E\left[s_{k} s_{l}\right]=(2 p-1) E\left[s_{k-1} s_{l}\right]
$$

extended to a more general case. Since $E\left[u_{k} u_{k}\right]=h^{2}$, we have:

with

$$
E\left[u_{k} u_{k+m}\right]=h^{2} q^{|m|}
$$

$$
q=2 p-1
$$

The covariance function of $u$ in equation (4) serves as the basis to find the frequency spectrum of the GBS (Tulleken, 1990). Considering the normalized case, $h=1$, we have

$$
\begin{aligned}
\Phi_{u}(\omega, q)= & T \sum_{m=-\infty}^{\infty} E\left[u_{k} u_{k+m}\right] \mathrm{e}^{-i m \omega T}=\frac{\left(1-q^{2}\right) T}{1-2 q \cos \omega T+q^{2}}, \\
& \omega \in[0, \pi / T],
\end{aligned}
$$

where $\Phi_{u}$ is the frequency spectrum of the input $u$ and it is termed as the input spectrum hereafter. It immediately becomes clear that when $p=0.5$ (or $q=0$ ) the input spectrum remains

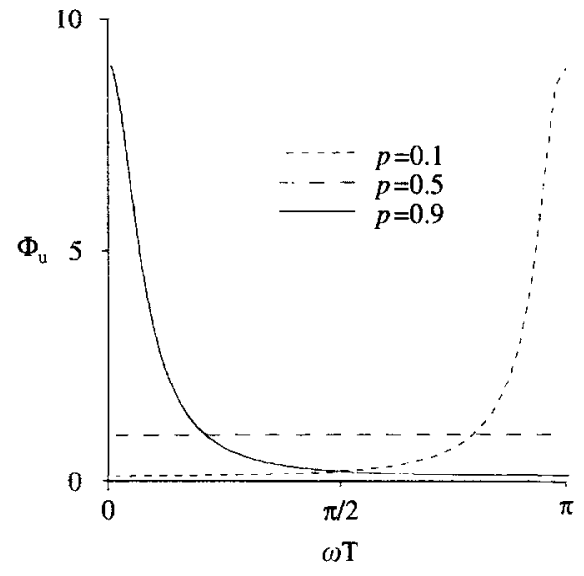

Fig. 2. Frequency spectra of GBS for different values of $p$.

constant from zero frequency up to the Nyquist frequency $\pi / T$ (Fig. 2). However, as $p>\frac{1}{2}$ the energy of the input signal is shifted toward the low-frequency range (Fig. 2) and the opposite behavior can be observed for the case of $p<\frac{1}{2}$. This additional degree of freedom provides an attractive alternative to place the emphasis on the frequency range of interest.

\section{Main results}

Despite establishing the fundamentals for the GBS, Tulleken (1990) addresses the issues of input design for the ARMA type of models, instead of relevant frequency ranges. Here, we are more interested in designing appropriate GBS as input signal when specifications are given on a particular frequency or certain frequency interval.

3.1. Single frequency point. Under the framework of the GBS, we would like to find the optimal non-switching probability, $p^{*}$, such that the maximum input spectrum is placed on the frequency of interest for $p \in[0,1]$. The following theorem calculates the given frequency to $p^{*}$.

Theorem 1. Consider a GBS with the basis switching time $T$ [equation (2)]. The non-switching probability gives the maximum input spectrum on a particular frequency $\omega, \omega \in[0, \pi / T]$, is:

$$
p=\frac{1}{1+\tan (\omega T / 2)}
$$

Proof. See Appendix A.

This theorem provides a quantitative assessment on how $p$ should be chosen when the emphasis is placed on a particular frequency point. For example, if the zero frequency, i.e. the steady-state behavior, is of interest, the non-switching probability becomes unity and a step input should be used (Fig. 1) and if the Nyquist frequency, $w=\pi / T$, is of interest, the non-switching probability becomes zero (Fig. 1) and a square wave is generated. Figure 3 shows the optimal $p, p^{*}$ for the GBS when the specification is given on a certain frequency point.

Despite the interesting result derived for the GBS at a singlefrequency point, in practice, the frequency-domain specifications are often given over a frequency range of interest. Therefore, it will be useful to find the optimal non-switching probability for the GBS if the specification is a frequency interval.

3.2.. Frequency interval. The following theorem gives the optimal non-switching probability for the GBS of which the maximum input energy is placed on a frequency interval of interest.

Theorem 2. Consider a GBS with the basic switching time $T$ [equation (2)]. The non-switching probability gives the 


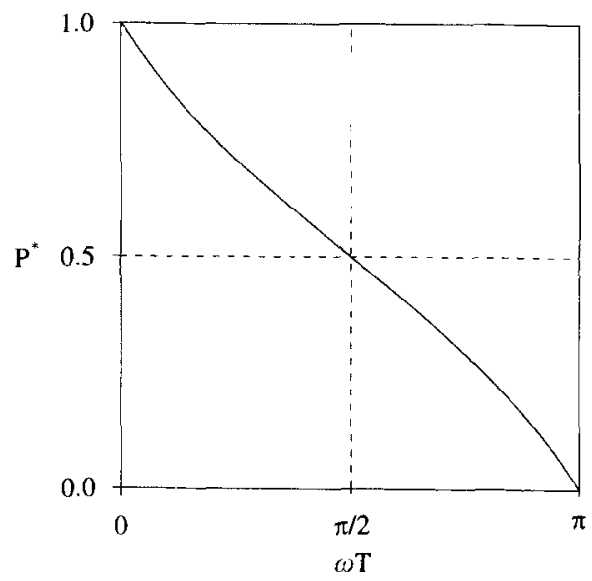

Fig. 3. Optimal non-switching probability for signal frequency point.

maximum input spectrum over a frequency interval $\left[\omega_{\mathrm{L}}, \omega_{\mathrm{H}}\right], \omega_{\mathrm{H}} \geq \omega_{\mathrm{L}}$ and $\omega_{\mathrm{L}}, \omega_{\mathrm{H}} \in[0, \pi / T]$, is:

$$
p=\frac{1}{1+\sqrt{\tan \left(\frac{\omega_{\mathrm{L}} T}{2}\right) \tan \left(\frac{\omega_{\mathrm{H}} T}{2}\right)}} .
$$

Proof. See Appendix B.

Actually, the result, equation (8), represents a generalization for the case of single frequency point. The second factor in the denominator of equation (8) is a geometric mean of the corresponding tangent functions and when $\omega_{\mathrm{H}}=\omega_{\mathrm{L}}$, equation (8) reduces to that of single-frequency point, equation (7).

The simple result of equation (8) forms the basis for the input design when the generalized binary sequence is employed. Therefore, the procedure for the input design with a frequencydomain specification expressed in terms of an interval $\left[\omega_{\mathrm{L}}, \omega_{\mathrm{H}}\right]$ can be summarized as follows:

S1. choose an appropriate input magnitude $h$;

S2. compute the optimal non-switching probability, $p^{*}$, from equation (8) for a given $\left[\omega_{\mathbf{L}}, \omega_{\mathbf{H}}\right]$;

S3. generate the input sequence, $u_{k}$, using $p^{*}$.

\section{Example}

Let us take a transfer function model to illustrate the proposed input design. Consider the following second order with dead-time system:

$$
G(s)=\frac{\mathrm{e}^{-s}}{(s+1)(10 s+1)}
$$

If we are interested in the frequency range of $[0.51,0.93]$ (corresponding to the following phase angle range $-135 \sim-180$ ), the non-switching probability can be calculated from equation (8) and we have

$$
p=\frac{1}{1+\sqrt{\tan (0.51 T / 2) \tan (0.93 T / 2)}} .
$$

For the case of $T=1$, we have $p^{*}=0.73$. A sequence of input (i.e. 200 points) is generated using $p^{*}=0.73$ with $h=1$ and the output is corrupted with Gaussian noise with a variance of 0.5 . The frequency response of the system is obtained from the Fourier transformation of the input-output data. Comparison is made between the GBS with different non-switching probability, $p=0.5,0.73$, and 0.95 . The results, Fig. 4 , show that the optimal GBS, i.e. $p=0.73$, gives better response.
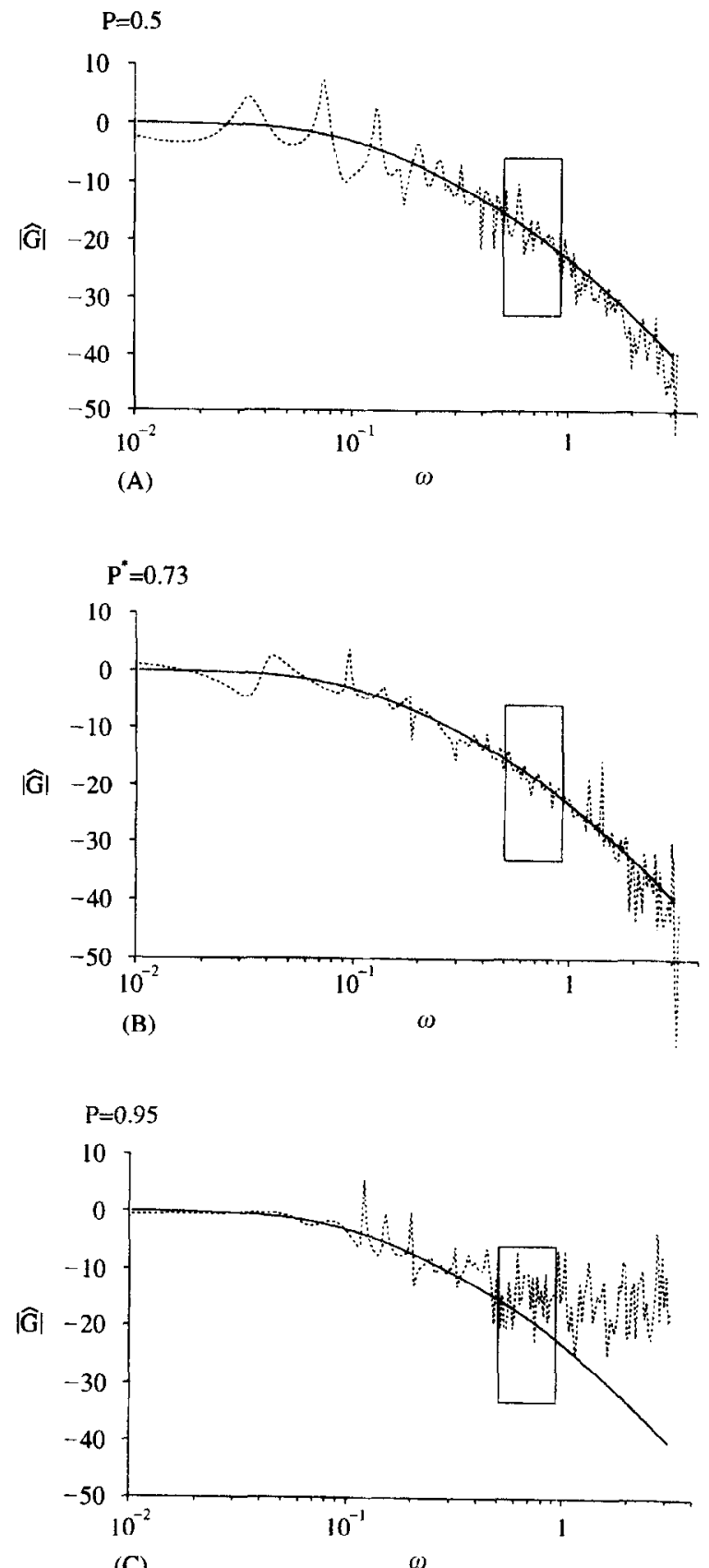

Fig. 4. Frequency responses of true (solid) and estimated (dashed) transfer functions using GBS-based input with different values of $p$.

\section{Conclusions}

The GBS of Tulleken has the advantage of shifting the distribution of frequency spectra. Therefore, it offers an attractive alternative in input design for system identification. In this work, analytical expressions are derived for the GBS when the frequency-domain specifications are given as a single-frequency point or a frequency interval. The simple results derived can be useful for experimental design of input sequence.

\section{References}

Bailey, J. K. (1995). Process identification using finite impulse response models. J. Process. Control, 5, 77-84.

Belforte, G. and T. T. Tay (1993). Optimal input design for worst-case system identification in $l_{1} / l_{2} l_{x x}$. System Control Lett., 20, 273-278. 
Chan, M. D. (1995). Identification of a simulated Shell distillation column using time series analysis method. J. Process Control, 5, 99-103.

Chien, I. L. (1996). Simple empirical nonlinear model for temperature-based high-purity column. A.I.Ch.E. J., 42, 149-162.

Cluett, W. R. and L. Wang (1991). Modelling and robust controller design using step response data. Chem. Engng. Sci., 46, 2065-2077.

Cumming, I. G. (1970). Frequency of input signal in identification. In 2nd IFAC Symp. on Identification, Prague.

Eykhoff, P. (1974). System Identification, Parameter and State Estimation, Wiley, London.

Goodwin, G. C. and R. L. Payne (1977). Dynamic System Identification: Experiment Design and Data Analysis, Academic Press, New York.

Ljung, L. (1987). System Identification: Theory for the User, Prentice-Hall, Englewood Cliffs, NJ.

Luyben, W. L. (1990). Process Modeling, Simulation and Control for Chemical Process, 2nd edn. McGraw-Hill, New York.

Marlin, T. E. (1995). Process Control: Designing Processes and Control System for Dynamic Performance, McGraw-Hill, New York.

Prett, D. M., T. A. Skrovanek and J. F. Pollard (1986). Process identification-past, present, future. In Shell Process Control Workshop, eds, Prett, D. M. and M. Morari, pp. 79-104. Butterworths, Boston, MA.

Schoukens, J., R. Pinteon, E. van der Ouderaa and J. Renneboog (1988). Survey of excitation signals for FFT based signal analyzer. IEEE Trans. Instrum. Measures, 37, 342-352.

Shen, S. H., J. S. Wu and C. C. Yu (1996). Use of biasedrelay feedback for system identification. $A I C h E J ., 42$, 1174-1180.

Sriniwas, G. R., Y. Arkun, I. L. Chien and B. A. Ogunnaike (1995). Nonlinear identification and control of a high-purity distillation column: a case study. J. Process. Control, 5, $149-162$.

Tulleken, H. J. A. F. (1990). Generalized binary noise test-signal concept for improved identification-experiment design. $\mathrm{Au}$ tomatica, 26, 37-49.

Tyreus, B. D. and W. L. Luyben (1992). Tuning PI controllers for integrator/dead time process. Ind. Engng. Chem. Res., 31, 2625-2628.

\section{Appendix $A$}

Since $\Phi_{u}(\omega, q)$ is convex for $\omega T \in[0, \pi]$ and $q \in[-1,1]$, the extreme value can be obtained by taking the derivative of the input specturm $\Phi_{\mathrm{u}}$ with respect to $q$.

$$
\frac{\mathrm{d} \Phi_{u}}{\mathrm{~d} q}=0 .
$$

From equation (6), we have

$$
q=\frac{1 \pm \sqrt{1-\cos \omega T}}{\cos \omega T}, \quad \omega \in[0, \pi / T]
$$

The definition of $q$ [equation (5)] indicates that $q$ ranges from -1 to 1 . Thus, only the minus sign in the above equation is valid. After some algebraic manipulation, the optimal nonswitching probability can then be expressed as

$$
p=\frac{1}{1+\tan \left(\frac{\omega T}{2}\right)}
$$

with $\omega T \in[0, \pi]$.

\section{Appendix $B$.}

For the case of a frequency intcrval, we would like to maximize the input power over a particular frequency range, i.e. $\left[\omega_{\mathrm{L}}, \omega_{\mathrm{H}}\right]$ with $\omega_{\mathbf{H}} \geq \omega_{\mathrm{L}}$ and $\omega_{\mathrm{L}}, \omega_{\mathbf{H}} \in[0, \pi / T]$. First, integrating $\Phi_{u}$ from $\omega_{\mathrm{L}}$ to $\omega_{\mathrm{H}}$ gives

$$
\begin{array}{r}
\int_{\omega_{\omega_{L}}}^{\omega_{\mathbf{H}}} \Phi_{u} \mathrm{~d} \omega=2 T\left\{\tan ^{-1}\left[\left(\frac{1+q}{1-q}\right) \tan \frac{\omega_{\mathbf{H}} T}{2}\right]\right. \\
\left.-\tan ^{-1}\left[\left(\frac{1+q}{1-q}\right) \tan \frac{\omega_{\mathbf{L}} T}{2}\right]\right\} .
\end{array}
$$

Taking the derivative of the integrand with respect to $q$ and letting it equals to zero, we arrive at

$$
\frac{1+q}{1-q}=\frac{1}{\sqrt{\tan \left(\frac{\omega_{\mathrm{L}} T}{2}\right) \tan \left(\frac{\omega_{\mathbf{H}} T}{2}\right)}}
$$

Substituting the definition of $p$ [equation (5)] into the equation, the optimal non-switching probability becomes

$$
p=\frac{1}{1+\sqrt{\tan \left(\frac{\omega_{L} T}{2}\right) \tan \left(\frac{\omega_{\mathrm{H}} T}{2}\right)}} .
$$

